\title{
A comparative pharmaco-metabolomic study of glutaminase inhibitors in glioma stem-like cells confirms biological effectiveness but reveals differences in target-specificity
}

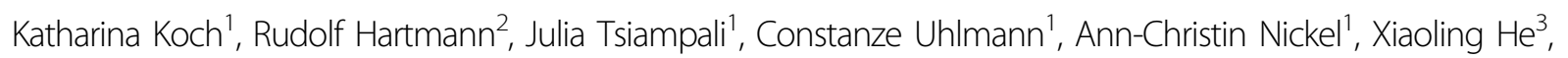
Marcel A. Kamp ${ }^{1}$, Michael Sabel ${ }^{1}$, Roger A. Barker ${ }^{3}$, Hans-Jakob Steiger ${ }^{1}$, Daniel Hänggi ${ }^{1}$, Dieter Willbold @id, Jaroslaw Maciaczyk $k^{1,5}$ and Ulf D. Kahlert ${ }^{1,6}$

\begin{abstract}
Cancer cells upregulate anabolic processes to maintain high rates of cellular turnover. Limiting the supply of macromolecular precursors by targeting enzymes involved in biosynthesis is a promising strategy in cancer therapy. Several tumors excessively metabolize glutamine to generate precursors for nonessential amino acids, nucleotides, and lipids, in a process called glutaminolysis. Here we show that pharmacological inhibition of glutaminase (GLS) eradicates glioblastoma stem-like cells (GSCs), a small cell subpopulation in glioblastoma (GBM) responsible for therapy resistance and tumor recurrence. Treatment with small molecule inhibitors compound 968 and CB839 effectively diminished cell growth and in vitro clonogenicity of GSC neurosphere cultures. However, our pharmaco-metabolic studies revealed that only CB839 inhibited GLS enzymatic activity thereby limiting the influx of glutamine derivates into the TCA cycle. Nevertheless, the effects of both inhibitors were highly GLS specific, since treatment sensitivity markedly correlated with GLS protein expression. Strikingly, we found GLS overexpressed in in vitro GSC models as compared with neural stem cells (NSC). Moreover, our study demonstrates the usefulness of in vitro pharmacometabolomics to score target specificity of compounds thereby refining drug development and risk assessment.
\end{abstract}

\section{Introduction}

Metabolic reprogramming of bioenergetic and biosynthetic processes is a key event in malignant transformation $^{1}$. Tumor cells can maintain high proliferation rates by enhanced uptake and metabolism of glucose and glutamine (Gln) thereby fueling the two main anaplerotic pathways providing tricarboxylic acid (TCA) cycle

\footnotetext{
Correspondence: UIf D. Kahlert (mail@ulf-kahlert.com)

${ }^{1}$ Neurosurgery Department, University Hospital Duesseldorf, 40225

Duesseldorf, Germany

${ }^{2}$ Institute of Complex Systems (ICS-6) Structural Biochemistry and JuStruct:

Juelich Center for Structural Biology, Forschungszentrum Juelich, 52425

Juelich, Germany

Full list of author information is available at the end of the article

These authors contributed equally: Jaroslaw Maciaczyk, UIf D. Kahlert

Edited by Maria Victoria Niklison Chirou
}

intermediates to boost oxidative phosphorylation and biomass production ${ }^{2}$. Since glycolysis and glutaminolysis are dysregulated in neoplastic cells and tissues due to epigenetic and mutational changes ${ }^{3-6}$, they are compelling therapeutic targets in cancer therapy. However, the clinical application of glycolysis inhibitors remains challenging mainly due to adverse effects ${ }^{7,8}$. Alternative strategies of metabolic interference in cancer therapy have recently emerged including the restriction of glutaminolytic activity. Mitochondrial glutaminase (GLS) catalyzes the hydrolytic deamidation of Gln to glutamate (Glu) in the first step of glutaminolysis. Subsequently, Glu is either metabolized to the TCA cycle intermediate alphaketoglutarate $(\alpha K G)$, which is then used as a nitrogendonor during the synthesis of several nonessential amino 
acids, or mediates redox homeostasis by increasing the production of the antioxidant glutathione (GSH). Since several oncogenes regulate GLS expression and many studies have shown that cancer cells are GLSdependent $^{3,9,10}$, GLS inhibitors (GLSi) (have been designed and evaluated in preclinical and clinical trials for brain tumors (trial ID: NCI-2018-00876) ${ }^{11-13}$. The specific and orally bioavailable small molecule inhibitor CB839 has been shown to effectively reduce viability, chemosensitivity, and induce apoptosis in several tumor entities including breast, ovarian, prostate, and lung cancer $^{14-17}$. Furthermore, compound 968 (C968), another small molecule GLS inhibitor, has been reported to impair tumor cell clonogenicity, viability, and growth ${ }^{18-23}$. Glioblastoma (GBM), the most aggressive malignant brain tumor, expresses high levels of GLS however their dependency on Gln metabolism remains controversial ${ }^{24}$. Several studies report that GBMs depend on glutaminolysis to sustain cellular viability and TCA cycle anaplerosis $^{4,25,26}$. However, others report that growth inhibition upon Gln withdrawal is cell line-specific and that resistant cell lines upregulate alternative metabolic routes to compensate for the lack of nutrient supply ${ }^{27}$. Here we analyze the effect of GLS inhibition on stem cellenriched GBM in vitro models (GBM stem-like cells; GSCs), which have been suggested to be responsible for the emergence of therapy resistance and tumor relapse ${ }^{28-31}$. Accumulating evidences indicate that GSCs are characterized by distinct metabolic reprogramming and interfering in this may be a suitable strategy to combat those highly therapy resistance population of cells ${ }^{32-34}$. We assessed the therapeutic potential of two prominent GLSi on GSCs in vitro and validated GLS as a target with low adverse effects. Furthermore, the pharmacometabolic characterization of our in vitro models upon compound exposure highlights the potential of this technology for the risk assessment of drug candidates.

\section{Results}

\section{GSC in vitro models recapitulate the glutaminase expression status of patient samples}

In order to assess the relevance of GLS in human GBM tumors, tumor bulk samples of seven primary GBMs were homogenized and subjected to immunoblotting for GLS protein. GLS was clearly expressed in all GBMs although expression varied markedly between tumors (Fig. 1a). That suggests, that the susceptibility of a patients GBM to GLS inhibition varies as well. Publicly available mRNA expression data showed that GLS is markedly overexpressed in the leading edge of the tumor and infiltrating tumor tissue (Fig. 1b) and it is thought that these tumor compartments promote GSC enrichment through a mechanism called epithelial-mesenchymal (EMT)-like transition ${ }^{35}$. Concisely, GLS has been reported to regulate the expression of EMT markers in colorectal cancer ${ }^{36,37}$. To assess GLS expression in stem cell-enriched GBM

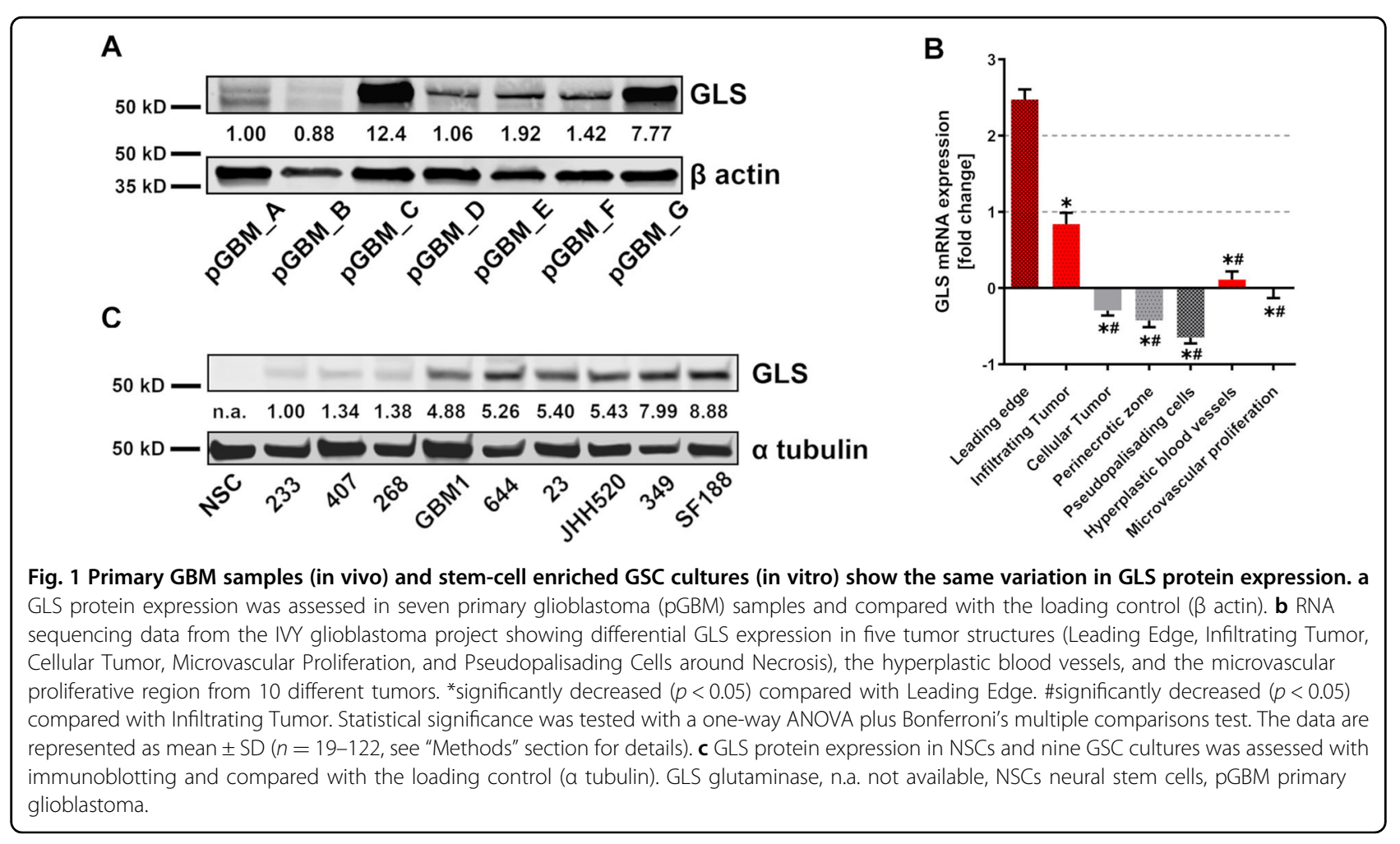


cultures, we performed immunoblotting for GLS protein in nine GSC cultures and compared the expression level to non-tumorigenic neural stem cells (NSCs) (Fig. 1c). Strikingly, we observed lowest GLS expression in NSCs and identified significant variations between the nine GSC cultures. Hence, we decided to study GLS suppression in six different GSC neurosphere cultures, three GLS-low (233, 407, 268) and three GLS-high (GBM1, JHH520, SF188) expressing ones. Importantly, our selected in vitro GSC models represent the physiological variation of GLS expression observed in tumor tissue therefore showing pathophysiological relevance (Fig. 1a). Verified by pixel densitometry analysis, the variation in GLS protein observed in the different primary tumors is similar to the variation in GLS expression observed between the different GSC in vitro models. For both protein analyses (Fig. 1a, c), the same experiment conditions were used and equal amounts of total protein were loaded.

\section{Susceptibility to pharamcological glutaminase inhibition correlates with elevated GLS expression}

To assess the effect of GLS inhibition on tumorigenic GSCs and estimate possible side effects on nontumorigenic stem cells, we treated highly clonogenic neurosphere cultures derived from human fetal cortical tissue (neural SC; NSC) with the small molecule GLSi C968 and CB839 and compared them to GLS-high (SF188, JHH520 and GBM1) and GLS-low (233, 407, and 268) expressing GSCs. Fetal NSCs are a commonly used surrogate for non-tumorigenic stem cells in brain tumor research ${ }^{38-41}$. Cell growth of GSC neurospheres and NSCs treated with increasing concentrations of C968 (Fig. 2a, b), CB839 (Fig. 2c, d), or vehicle (DMSO) was assessed after two and four days of incubation. In SF188, JHH520 and GBM1 cells, treatment with both C968 and CB839 induced a dose-dependent suppression of cell growth. On the contrary, 233, 407, and 268 showed no effects to GLS inhibition except for a moderate response in 407 to C968 and 268 to CB839 exposure. Nontumorigenic NSCs were resistant to both C968 and CB839 treatment.

\subsection{Glutaminase inhibition selectively impairs the stemness phenotype of GLS-high expressing cultures}

Since GSCs maintain their self-renewal ability they are capable of growing colonies out of single cells. Therefore, we tested the in vitro clonogenicity of GSCs upon treatment with $10 \mu \mathrm{M} \mathrm{C968} \mathrm{(Fig.} \mathrm{3a),} 1 \mu \mathrm{M} \mathrm{CB839}$ (Fig. 3b), or vehicle and compared them to non-tumorigenic NSCs. Consistent with the results from the growth assays, SF188, JHH520, and GBM1 cells showed markedly diminished clonogenic capacity, while the in vitro clonogenicity of GLS-low expressing cells (233, 407, and 268) was not affected except for a moderate reduction in 407 cells treated with C968. We can therefore directly correlate resistance to GLS inhibition with low protein expression levels (Fig. 1c). Consistent with our results, both pharmacological and genetic suppression of GLS protein caused impaired clonogenic capacity in different tumor entities ${ }^{17,23,42,43}$. Although reaching statistical significance, the in vitro clonogenicity was barely reduced in NSCs treated with $1 \mu \mathrm{M}$ CB839 and moderately reduced by $10 \mu \mathrm{M}$ C968 treatment (Fig. 3a, b). The observed effects were much less severe than observations in previous studies testing Gln analogs or genetic GLS suppression in non-tumorigenic $\mathrm{NSCs}^{44,45}$. To our knowledge, our study is the first risk assessment of targeted pharmacological GLS inhibition in NSCs. Again, the superiority of CB839 as a GLSi becomes obvious, showing less side effects in NSCs. Previous research directly correlated the clonogenic capacity of GSCs with the expression of GSC stemness marker prominin (CD133) and EMT activator ZEB1 $1^{31,35,46-48}$. Immunoblotting analyses should show whether the impaired clonogenicity we observed in treated GLS-high expressing GSCs (SF188, JHH520, GBM1) coincides with reduced CD133 or ZEB1 expression. Both C968 (Fig. 3c) and CB839 (Fig. 3d) treatment mildly reduced ZEB1 expression both in sensitive (GLS-high) and insensitive (GLS-low) GSCs. Furthermore, CD133 expression was dramatically reduced by C968 and moderately reduced by CB839 treatment independent of GLSi sensitivity. Since we observed similar effects in both sensitive and insensitive GSCs, our results clearly show that the degree of stemness in GBMs cannot be exclusively estimated by the expression levels of single markers like CD133 or ZEB1, but should rather be defined by phenotypical readouts like the in vitro clonogenicity.

\section{Glutaminase inhibition causes cell cycle arrest in sensitive GLS-high expressing GSCs}

Since functional glutaminolysis is a prerequisite for a variety of cellular processes, we assessed apoptosis induction, reactive oxygen species (ROS) accumulation, GSH concentrations and GSH/GSSG ratios, and the cell cycle state in GSCs treated with $10 \mu \mathrm{M}$ C968 or $1 \mu \mathrm{M}$ CB839. For those mechanistic studies we chose two GLShigh (JHH520 and GBM1) and two GLS-low (407 and 233) expressing GSC cultures. In GLS-high expressing cells, treatment with both inhibitors significantly increased the percentage of cells in quiescent G0/G1 phase and reduced the percentage of cells in the proliferative $\mathrm{S}$ and M/G2 phases (Fig. 4a). However, treatment with C938 and CB839 did not significantly alter the cell cycle of GLS-low expressing 233 or 407 cells. Furthermore, we calculated the proliferation index as a measure of cells in proliferative cell cycle phases $(\mathrm{PI}=(\mathrm{S}$ $+\mathrm{G} 2 / \mathrm{M}) /(\mathrm{G} 0 / \mathrm{G} 1+\mathrm{S}+\mathrm{G} 2 / \mathrm{M}) \times 100 \%)$. We observed a significant decrease of the PI in JHH520 (-28\%), GBM1 


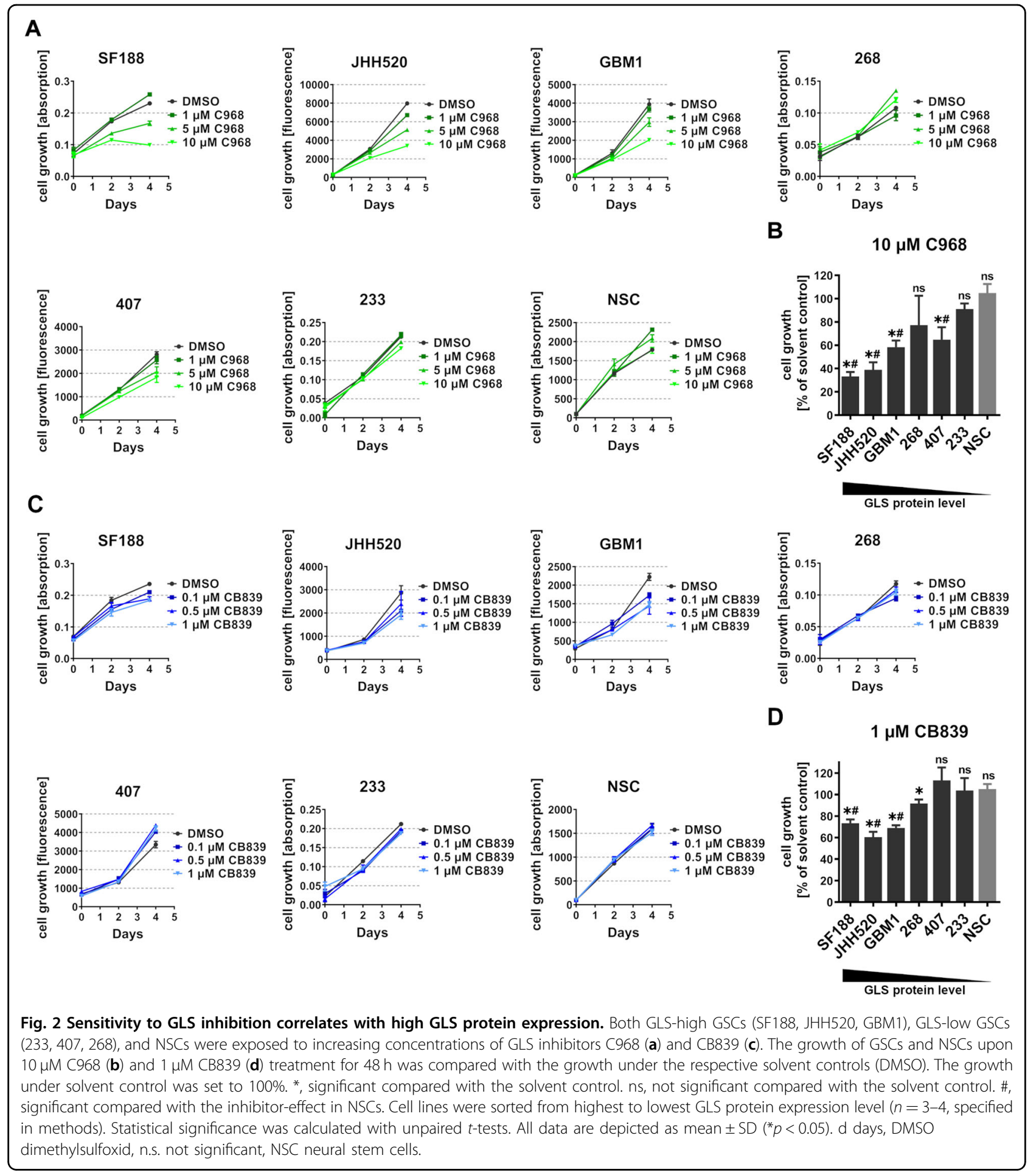

$(-11 \%)$, and $407(-11 \%)$ upon C968 treatment and in JHH520 (-24\%) and GBM1 (-18\%) cells upon CB839 treatment. In lowest GLS expressing 233 cells neither C968 nor CB839 affected the PI. Furthermore, we costained GSCs treated with GLSi for apoptotic cells with AnnexinV and 7-AAD. FACS measurements revealed no significant increase in the percentages of early apoptotic (EA, AnnexinV $V^{\text {pos }}, 7-A_{A D}{ }^{\text {neg }}$ ) or late apoptotic (LA, AnnexinV $V^{\text {pos }}$, 7-AAD ${ }^{\text {pos}}$ ) cells upon C968 or CB839 treatment (Fig. 4b). However, when we normalized the percentages of total apoptotic cells (EA + LA) in the treatment conditions to the respective solvent controls we 


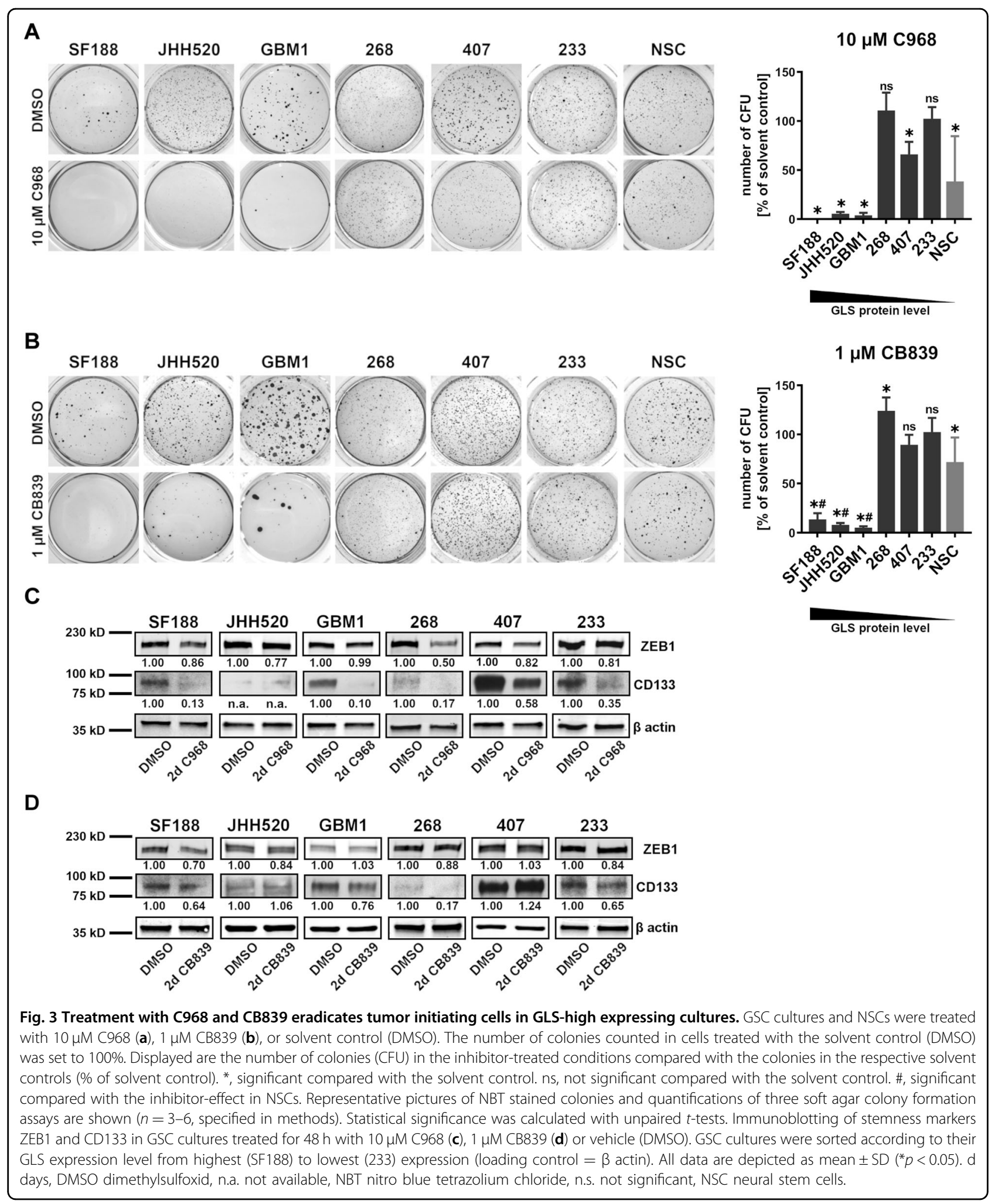

observed a significant increase in total apoptotic cells by 1.99-fold in JHH520, 1.28-fold in GBM1, and 1.23-fold in 407 cells treated with C968, but saw no effect in 233 cells
(Fig. 4c). CB839 treatment did not significantly induce apoptosis in any of the cell lines. Since the GLS product Glu is crucial for the synthesis of the antioxidant GSH, we 


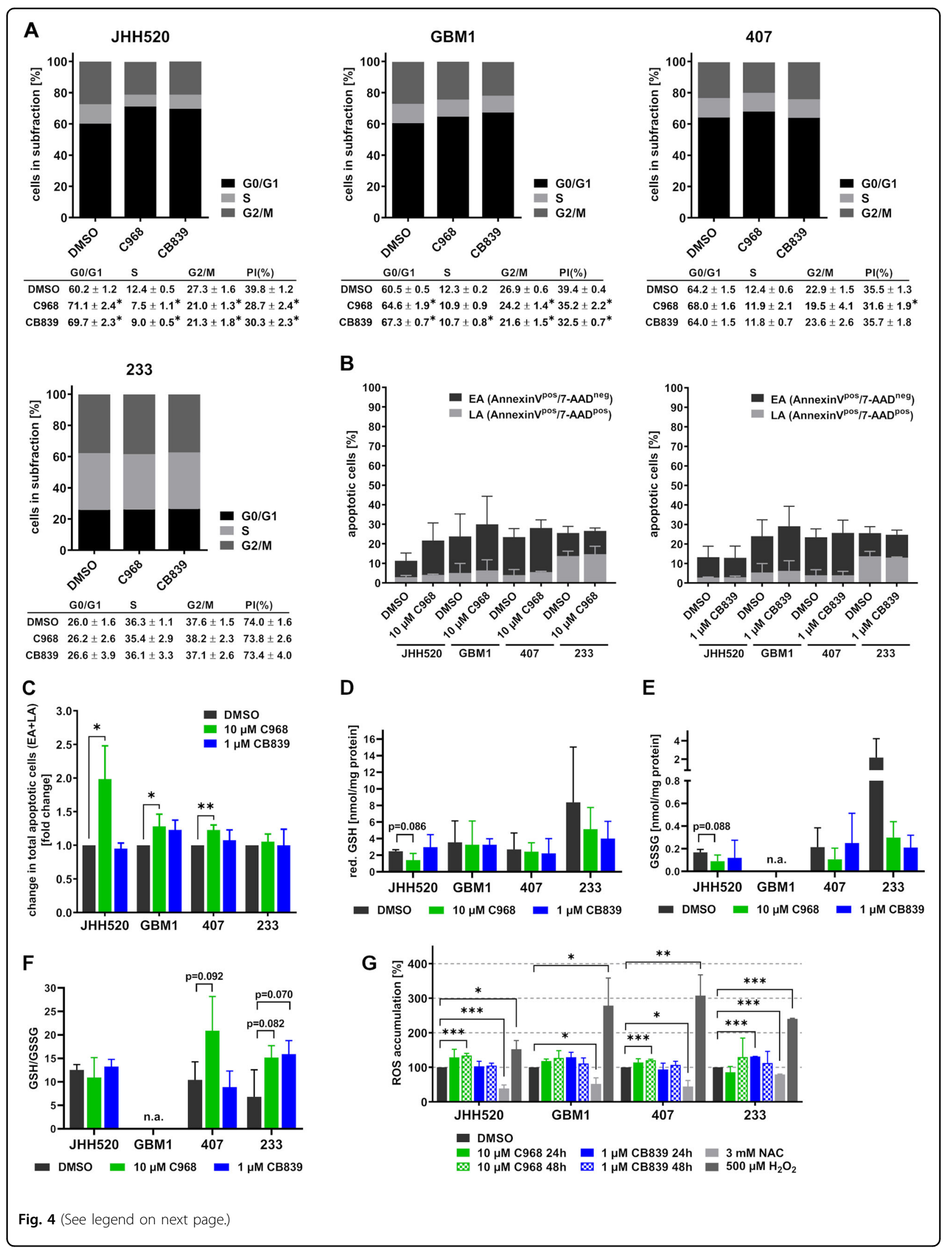


(see figure on previous page)

Fig. 4 CB839 selectively causes cell cycle arrest in GLS-high expressing cultures without inducing apoptosis or oxidative stress. a

Percentage of cells in quiescent (G0/G1), synthesis (S), or mitosis (G2/M) phase after treatment with $10 \mu \mathrm{M} \mathrm{C968,1 \mu M}$ CB830, or vehicle (DMSO) for $48 \mathrm{~h}$. The proliferation index $(\mathrm{PI}=(\mathrm{S}+\mathrm{G} 2 / \mathrm{M}) /(\mathrm{GO} / \mathrm{G} 1+\mathrm{S}+\mathrm{G} 2 / \mathrm{M}) \times 100 \%)$ was calculated to visualize the percentage of proliferative cells. Statistical significance was tested with two-way ANOVA $(n=3)$. b The percentages of early apoptotic (EA, AnnexinV $V^{\text {pos }}, 7-A A D^{\text {neg }}$ ) and late apoptotic $(L A$, AnnexinV ${ }^{\text {pos }}, 7-A A D^{\text {pos}}$ ) cells in GSC cultures treated for $48 \mathrm{~h}$ with $10 \mu \mathrm{M}$ C968 and $1 \mu \mathrm{M}$ CB839 were assessed with flow cytometry. c Fold changes in total apoptotic cells (EA + LA) of C968- and CB839-treated GSCs compared with cultures treated with DMSO ( $n=3$ for C968, $n=4$ for CB839). Reduced (GSH) [nmol/mg protein] (d) and oxidized (GSSG) [nmol/mg protein] (e) glutathione was measured in GSCs treated with $10 \mu \mathrm{M}$ C968, $1 \mu \mathrm{M}$ CB830, or vehicle (DMSO) for $48 \mathrm{~h}$. $\mathbf{f}$ The ratio of reduced (GSH) and oxidized (GSSG) glutathione in GSCs treated with $10 \mu \mathrm{M} \mathrm{C968,1 \mu M} \mathrm{CB830,} \mathrm{or}$ vehicle (DMSO) for $48 \mathrm{~h}(n=3)$. g ROS accumulation was measured via DCFDA oxidation over $24 \mathrm{~h}$ and $48 \mathrm{~h}$ in cells treated with either $10 \mu \mathrm{M} C 968$, $1 \mu \mathrm{M} \mathrm{CB830}$, or DMSO. Exposure to $1 \mathrm{mM} \mathrm{NAC}$ was used as the negative and exposure to $500 \mu \mathrm{M} \mathrm{H}_{2} \mathrm{O}_{2}$ as the positive control $(n=3)$. If not specified, statistical significance was calculated with unpaired $t$-tests. All data are depicted as mean \pm SD $\left({ }^{*} p<0.05,{ }^{* *} p<0.01,{ }^{* * *} p<0.001\right)$. DMSO

dimethylsulfoxid, GSH glutathione, n.a. not available, NAC N-acetylcysteine, n.s. not significant, ROS reactive oxygen species.

assessed whether GLS inhibition alters the concentrations of reduced (GSH) or oxidized (GSSG) glutathione or affects the GSH/GSSG ratio. Decreased GSH/GSSG ratios are a measure of oxidative stress in vitro and in vivo ${ }^{49}$. Except for a nearly significant reduction of GSH $(p=$ $0.086)$ and GSSG $(p=0.088)$ concentrations in C968treated JHH520 cells, we observed no significant effects on the GSH or GSSG concentrations (Fig. 4d, e). Interestingly, the GSH/GSSG ratio was even increased in insensitive GLS-low 407 ( $p=0.092$ for C968) and 233 $(p=0.082$ for C968 and $p=0.070$ for CB839) cells treated with GLSi (Fig. 4f). In GBM1 cells, we could not detect GSSG and thus could not calculate the GSH/GSSG ratio. This is not uncommon, since reduced GSH makes up on average $98 \%$ of the total GSH content ${ }^{49}$. Since alterations in antioxidant levels affect the intracellular clearance of ROS, we assessed ROS accumulation after treatment with $10 \mu \mathrm{M} \mathrm{C} 968$ or $1 \mu \mathrm{M}$ CB839 for 24 and $48 \mathrm{~h}$. Except for a $30 \%$ increase in ROS accumulation in 233 cells treated with CB839 for $24 \mathrm{~h}, \mathrm{CB} 839$ treatment did not induce a significant increase in intracellular ROS (Fig. 4g). Furthermore, $48 \mathrm{~h}$ C968 exposure increased ROS levels in GLS-high JHH520 (34\%) and GLS-low 407 (20\%) cells. The ROS inhibitor N-Acetyl-L-cysteine (NAC) significantly reduced and $\mathrm{H}_{2} \mathrm{O}_{2}$ significantly increased ROS accumulation. Consistent with our previous findings, these results further emphasize the selective mode of action of GLSi on cells with abundant GLS protein expression, since especially the observed effects on viability, clonogenicity, cell cycle, and apoptosis were most pronounced in GLS-high expressing cells.

\section{Glutaminase inhibition with CB839 targets the anabolism of GSCs by diminishing the influx into the TCA cycle}

To see whether metabolic changes cause the phenotypic effects of GLS inhibition, we used high resolution proton magnetic resonance spectroscopy (HR ${ }^{1} \mathrm{H}$ NMR) to analyze intracellular metabolites of JHH520, GBM1, 407, and 233 cells treated with $10 \mu \mathrm{M}$ C968 or $1 \mu \mathrm{M}$ CB839. Surprisingly, only CB839 treatment induced the anticipated changes in GSC metabolites. Blockade of GLS activity by CB839 led to a significant reduction of the enzyme product (Glu), accumulation of the educt (Gln), and decreased the Glu/Gln ratio in all four cell lines (Fig. 5a, b). This metabolic phenotype has been validated in several in vitro and in vivo studies as a reproducible measure of GLS inhibition ${ }^{14,50,51}$. Strikingly, upon treatment with C968 we neither observed reduced product (Glu) concentrations nor increased educt (Gln) concentrations, except for a moderate Glu reduction in 233 cells. Even in most sensitive JHH520 cells, C968 treatment did not decrease the Glu/Gln ratio. Since Wang et al. showed efficient GLS inhibition with $20 \mu \mathrm{M}$ C968 in hepatocellular carcinoma cells which do not respond to $10 \mu \mathrm{M}$ $\mathrm{C} 968^{20}$, we additionally measured the GLS activity in GSC cultures treated with $20 \mu \mathrm{M}$ C968. However, treatment of our GSC cultures with $20 \mu \mathrm{M}$ C968 did not alter the Glu/ Gln ratio and therefore did not inhibit the GLS activity. Furthermore, CB839, but not C968, reduced intracellular concentrations of the TCA cycle intermediate succinate (Suc) and Glu-dependent amino acids aspartate (Asp) and alanine (Ala) in all four cell lines. We further analyzed levels of known oncometabolites upon treatment with C968 and CB839 to see whether GSCs regulate other metabolic pathways that may compensate for glutaminolysis inhibition. In sensitive (JHH520, GBM1) but not insensitive $(407,233)$ cells CB839 treatment increased choline metabolism leading to elevated levels of phosphocholine and total choline. In contrast, C968 treatment had no consistent effect on GSC oncometabolites. Since we observed reduced Suc, Asp, and Ala levels upon CB839 treatment, we hypothesized that insufficient supply of TCA cycle intermediates after GLS inhibition disturbs the anabolism of GSCs causing a decline in the GSC pool. To test this hypothesis, we tried to rescue the anti-growth effect of C968 and CB839 by addition of either $4 \mathrm{mM}$ Glu or $4 \mathrm{mM} \alpha \mathrm{KK}$. The C968 treatment could neither be rescued by Glu nor $\alpha \mathrm{KG}$ (Fig. $5 \mathrm{c}$ ). In contrast, the antigrowth effect of CB839 in sensitive JHH520 and GBM1 cells could be efficiently rescued by both addition 


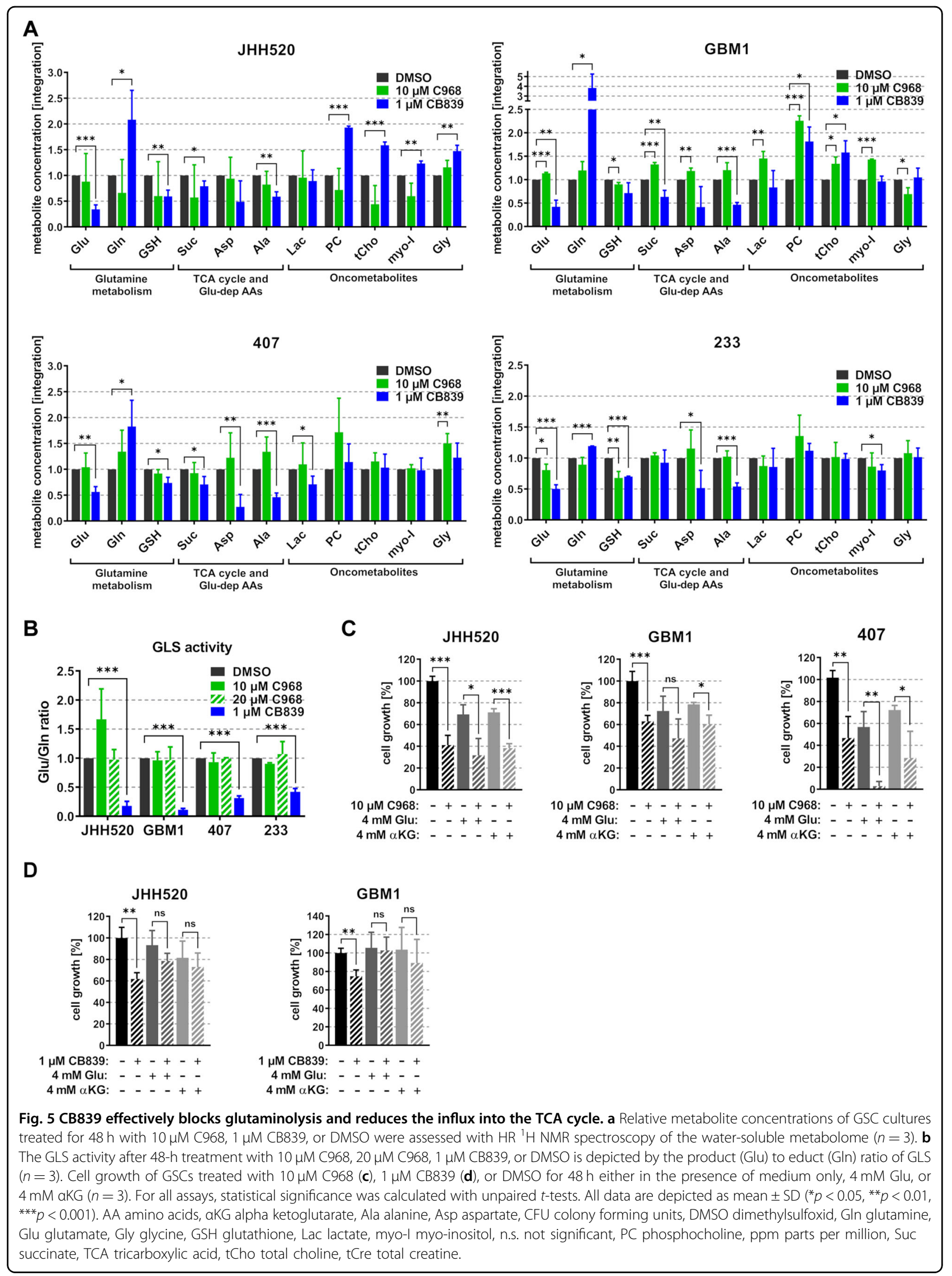


of Glu and $\alpha \mathrm{KG}$ (Fig. $5 \mathrm{~d}$ ), indicating that CB839 eradicates GSCs by diminishing TCA-cycle dependent processes necessary for GSC maintenance and cell cycle progression. Consistent with our results, previous studies reported that the maintenance of cancer stem-like cells depends on a high flux rate through the TCA cycle ${ }^{52,53}$ and that cell cycle progression in cells is limited by the availability of biosynthetic precursors from the TCA cycle ${ }^{54,55}$. Our ${ }^{1} \mathrm{H}$ NMR data, clearly show that C968 fails to suppress glutaminolysis, since we neither observed a reduction in the GLS product Glu, nor any downstream products of Glu such as Suc, Asp, or Ala upon C968 treatment (Fig. 5a, b). Interestingly, CB839 also induced metabolic changes in insensitive 407 and 233 cells. However, the effects were less marked than those observed in GLS-high expressing JHH520 and GBM1 cells. We hypothesize that due to the low GLS baseline expression, 407 and 233 cells are less dependent on functional GLS.

\section{Discussion}

Functional glutaminolysis is crucial for the bioenergetic and biosynthetic homeostasis especially in proliferative and invasive cancer cells. Here we show that maintenance of highly aggressive GSCs markedly depends on intracellular Glu synthesis by GLS and that this dependency strongly correlates with GLS protein expression. We show that GLS is expressed in GBM tumors and its level of expression greatly varies among patients (Fig. 1a). In a previous study on matching control brain and tumor samples from GBM patients, it was further shown that tumors express elevated GLS protein levels ${ }^{26}$. Moreover, increased GLS expression has been correlated with higher grade brain cancers, shortened patient survival, and temozolomide (TMZ) resistance ${ }^{24}$. In this manuscript we show elevated GLS transcription in the invasive front of GBMs, known to contain highly invasive GSCs ${ }^{35}$. Previous publications reported that genetic and pharmacological inhibition of GLS attenuates stemness properties in hepatocellular, colorectal, and prostate cancer $^{21,23,43}$. Furthermore, our group showed that the prominent route of anti-GSC therapy using $\gamma$-secretase inhibitor MRK003 targets GSC growth, in part by reducing intracellular Glu as a consequence of GLS inhibition ${ }^{56}$.

Here we correlate the resistance to GLS inhibition with reduced protein levels of GLS showing strong phenotypical consequences in high expressing GSC cultures (SF188, JHH520, GBM1) but only mild in low expressing GSCs $(233,407,268)$ or non-neoplastic NSCs (Figs. 2, 3). This is in line with similar observations in triple-negative breast cancer $(\mathrm{TNBC})^{57}$. Furthermore, the observed anti-stemness effect of GLS inhibition has been reported by several studies highlighting impaired clonogenicity as a major phenotype of pharmacological and genetic GLS suppression ${ }^{23,43,58}$.
Recent studies reported that targeted GLS inhibition further causes cell cycle arrest in prostate and non-small cell lung cancer ${ }^{19,43}$. This is consistent with our results showing cell cycle arrest in G0/G1 and a reduced fraction of cells in S- and M-phase after treatment with C968 (JHH520, GBM1, 407) and CB839 (JHH520 and GBM1) (Fig. 4). Again, especially the effectiveness of CB839 treatment correlated with the GLS expression level. Clonogenic GSCs make up just around 10\% of our cultures (max. 10\% of plated cells form colonies, Fig. 3), therefore the observed effects on cell cycle could explain the profound effects on clonogenicity if we assume that GSCs are especially affected by GLS inhibition.

Off-target effects are a common problem of many prominent cancer drug candidates in clinical trials ${ }^{59}$. Given the increasing attention of GLSi in various cancer trials, we sought to probe the target specificity of two leading GLSi compounds with the focus on their functional effects on malignant and nonmalignant stem cells. We chose C968 given its reproducibly reported therapeutic potential in many cancer studies including our own $^{19,21,22}$, and CB839, as one of the leading clinical GLSi compounds in oncology (trial IDs: NCI-2018-00876, NCI2019-01365, NCI-2019-00572). To our knowledge, no study has explicitly addressed the metabolic consequences of these compounds in functional assays which score their therapeutic effects preferentially as a consequence of effective target suppression. Surprisingly, our pharmacometabolic studies revealed that C968 treatment neither increased intracellular Gln, nor did it reduce the concentrations of Glu, Glu-dependent amino acids (Ala, Asp) or the TCA cycle intermediate Suc (Fig. 5), all validated metabolic indicators for successful GLS suppres$\operatorname{sion}^{51,57,60}$. This suggests that the phenotypes observed upon C968 treatment (Figs. 2, 3) cannot be explained by glutaminolysis suppression. This is supported by our observations that the effect of C968 treatment could neither be rescued by addition of Glu nor by replenishing the TCA cycle with $\alpha \mathrm{KG}$. This strongly suggests that C968 does not affect the enzymatic activity of GLS in GSCs. On the other hand, since sensitivity towards C968 treatment positively correlates with elevated GLS protein expression levels (Figs. 2, 3), the effects cannot solely be explained by non-specific cytotoxicity and application of metabolic flux analyses with isotope-labeled Gln are needed to pin-point the mode of action of this drug candidate.

In contrast, CB839 effectively reduced intracellular Glu concentration while causing accumulation of Gln (Fig. 5). Furthermore, CB839 diminished the influx into the TCA cycle and interfered with GSC anabolism by markedly reducing the levels of Suc, Ala, and Asp. These observations are in line with several pharmaco-metabolic studies showing Gln accumulation and decreased Glu, GSH, and Asp concentrations upon CB839 treatment ${ }^{14,61}$. Previous 
studies have reported that cancer stem-like cells depend on a high flux rate through the TCA cycle ${ }^{52,53}$, suggesting that CB839 treatment diminishes the GSC population by reducing the influx of Glu into the TCA cycle. Indeed, both addition of Glu and $\alpha \mathrm{KG}$ effectively rescued the phenotype caused by CB839 treatment (Fig. 5). This is in line with previous research showing that upregulation of aKG-dependent Gln metabolism and increased GLS expression promotes the maintenance of cancer stem cells through various mechanisms ${ }^{26,62-65}$. Furthermore, Gln metabolism promotes the maintenance of stemness through elevating the synthesis of GSH and maintenance of a balanced redox homeostasis ${ }^{23,66,67}$. However, we could neither detect significant accumulation of ROS nor decreases in the GSH/GSSG ratios in GSCs treated with CB839 (Fig. 4). Therefore, we conclude that CB839 predominantly diminishes the GSC pool by disrupting the influx of Glu into the TCA cycle, thereby limiting the bioenergetic and biosynthetic supply. Several studies describe a checkpoint in the late G1 phase of the cell cycle where the progress into $\mathrm{S}$ phase depends on the availability of precursors for nucleotide biosynthesis ${ }^{54,55}$. Therefore, reduced influx into the TCA cycle could explain the observed cell cycle arrest in G0/G1 phase upon treatment with CB839.

Taken together, our study clearly shows the potential of in vitro pharmaco-metabolomics for therapy efficacy scoring and risk assessment of compounds. Focusing on GBM and their therapy-resistant stem cell subpopulation, we further highlight the relevance of GLS as a druggable and promising therapeutic target in our need to improve the management of GBM therapy resistance and tumor relapse. Although new platforms for computational drug target discovery using molecular and cellular data of tumor material enable high throughput drug design and therapy resistance prediction $^{68}$, functional assays to biologically confirm computational biology findings are fundamental for the translational value of drug development and toxicology risk assessment. We found that CB839 significantly outperforms C968 in terms of enzymatic inhibitory potential and would be the preferred pharmacologic intervention when aiming at targeting glutaminolysis. CB839 shows effective GLS inhibition at low $\mu \mathrm{M}$ concentrations $(1 \mu \mathrm{M})$ whereas even high concentrations of C968 $(20 \mu \mathrm{M})$ do not affect GLS enzymatic activity. Our in vitro studies with non-transformed cells highlight the potential of CB839 as a cancer-specific precision treatment. Furthermore, our lab is aiming to improve the cancer cell specificity of CB839 and further reduce off-target effects by preferentially directing the delivery of the substance to cancer cells using nanotechnology engineering in a similar approach as recently described ${ }^{69}$.

\section{Materials and methods}

\section{Cell cultures and primary tissue specimen}

Glioma cell line JHH520 was generously provided by G. Riggins (Baltimore, USA), GBM1 by A. Vescovi (Milan, Italy), cell lines 23, 233, 268, 349, and 407 by M.S. Carro (Freiburg, Germany), cell line SF188 by E. Raabe (Baltimore, USA), and cell line NCH644 (644) by C. HeroldMende (Heidelberg, Germany). GSC neurospheres were cultured in DMEM w/o pyruvate (Gibco, \#11965092, Thermo Fisher Scientific, USA), 30\% Ham's F12 Nutrient Mix (Gibco, \#11765047), 2\% B27 supplement (Gibco, \#17504044), $20 \mathrm{ng} / \mathrm{ml}$ human bFGF (Peprotech, \#AF-10018B, USA), $20 \mathrm{ng} / \mathrm{ml}$ human EGF (Peprotech, \#AF-10015), $5 \mu \mathrm{g} / \mathrm{ml}$ Heparin (Sigma, \#H0878, Merck KGaA, Germany), and $1 \times$ Anti-Anti (Gibco, \#15240096). All cells were cultured at $37{ }^{\circ} \mathrm{C}$ and $5 \% \mathrm{CO}_{2}$. Cell lines were regularity tested for mycoplasma contamination and STR analyses were performed to guarantee authenticity and purity. Human fetal cortical tissue was collected in Cambridge UK under full ethical approval and sent to us where it was then transferred into cell culture after mechanic dissociation of cells. The cultures were enriched for NSCs by propagation in the above described neurosphere medium. Neurospheres were passaged by mechanical chopping with a Mcllwan Tissue Chopper (Campden Instruments, UK) every week. The study was conducted in accordance with the Declaration of Helsinki, and the protocol was approved by the Ethics Committee of the Medical Faculty of the Heinrich-Heine University (Study ID \#5206). Primary GBM tumor samples were derived from the operating theater at the department of neurosurgery (Duesseldorf, Germany) and were snap frozen in liquid nitrogen until the preparation of lysates was undertaken. All subjects gave their informed consent for inclusion before they participated in the study. The study was conducted in accordance with the Declaration of Helsinki, and the protocol was approved by the Ethics Committee of the Medical Faculty of the Heinrich-Heine University (\#2019-484-FmB).

\section{GLS inhibitors}

For GLS inhibition we used the small molecule inhibitors C968 (5-[3-bromo-4-(dimethylamino)phenyl]-2,3,5,6-tetrahydro-2,2-dimethyl-benzo[a]phenanthridin- $4(1 \mathrm{H})$-one, Merck, Germany, \#352010) and CB839 (N-[5-[4-[6-[[2-[3(trifluoromethoxy)phenyl]acetyl]amino]-3-pyridazinyl] butyl]-1,3,4-thiadiazol-2-yl]-2-pyridineacetamide, Cayman Chemicals, USA, \#22038). Stock solutions were prepared in dimethylsulfoxid (DMSO) and stored at $-20^{\circ} \mathrm{C}$.

\section{Western blotting}

Cell lysates were electrophoretically separated by SDS PAGE and transferred onto nitrocellulose membranes as described previously ${ }^{70}$. Primary antibodies against CD133 
(1:100, Miltenyi, Germany, \#W6B3C1), SOX2 (1:1000, Cell Signaling Technology, UK, \#L1D6A2), GLS (1:1000, Abcam, UK, \#ab93434), ZEB1 (1:2000, Sigma, \#HPA027524), $\beta$ actin (1:5000, Cell Signaling Technology, \#4970) and $\alpha$-tubulin (1:10,000, Sigma, \#T9026) were incubated overnight at $4{ }^{\circ} \mathrm{C}$ in $5 \%$ milk powder in Trisbuffered saline with $0.1 \%$ Tween-20 (TBST). The secondary antibodies goat-anti-rabbit IRDye $800 \mathrm{CW}$ (1:10,000, LI-COR, USA, \#926-32211), goat-anti-mouse IRDye680RD (1:10000, LI-COR \#926-68070), and goat anti-mouse-HRP $(1: 10,000$, Jackson ImmunoResearch, UK, \#111-035-003) were diluted in 5\% milk powder in TBST and incubated for $1 \mathrm{~h}$ at room temperature. Chemiluminescent signals were detected on a filmbased system using chemiluminescent substrates (Thermo Scientific, \#34096). Fluorescence-labeled antibodies were detected with a LI-COR Odyssey CLx Imager (LI-COR). Densitometry was performed with supplied software from LI-COR or ImageJ software ${ }^{71}$. For protein analysis of primary tumor samples, tissues were homogenized in RIPA lysis buffer using a $1 \mathrm{ml}$ Dounce Homogenizer. Lysates were then incubated for $45 \mathrm{~min}$ on ice and centrifuged at 13,000 rpm to yield the cleared lysate. For the immunoblot in Fig. 1c, we rearranged the individual lanes from lowest to highest GLS expression to improve visualization of the different expression patterns. All unprocessed pictures can be found in supplementary fig. 1. The remaining blots (Figs. 1, 3c, d) were not cut vertically. For all western blots, individual genes were tested on the same samples on the same membrane.

\section{Dual-phase metabolite extraction}

Water soluble metabolites were extracted as previously described $^{70,72,73}$. In brief, a minimum of $5 \times 10^{6}$ cells per sample were harvested, washed with PBS, and extracted with the dual-phase methanol/chloroform/water (1:1:1, $\mathrm{v} / \mathrm{v} / \mathrm{v})$ method. The cells were washed twice with $5 \mathrm{ml}$ ice-cold $0.9 \mathrm{mM} \mathrm{NaCl}$, resuspended in $850 \mu$ lice-cold $\mathrm{ddH}_{2} \mathrm{O}$ and transferred into pre-chilled glass tubes. After addition of $4 \mathrm{ml}$ of ice-cold methanol the tubes were vortexed vigorously and incubated on ice for $15 \mathrm{~min}$. Then $4 \mathrm{ml}$ of ice-cold chloroform was added, vortexed, and incubated for $10 \mathrm{~min}$ on ice. Finally, $3.15 \mathrm{ml}$ of ice-cold $\mathrm{ddH}_{2} \mathrm{O}$ was added, vortexed, and incubated overnight at $4{ }^{\circ} \mathrm{C}$. The samples were centrifuged for $30 \mathrm{~min}$ at $4{ }^{\circ} \mathrm{C}$ and $4500 \mathrm{rpm}$. The upper water-methanol phase was separated and incubated for 10 min with $10 \mathrm{mg}$ Chelex $^{\circledR} 100$ resin (Sigma, \#C7901) on ice. The samples were filtered through a $70 \mu \mathrm{m}$ mesh and the methanol was evaporated for $1 \mathrm{~h}$ at $30^{\circ} \mathrm{C}$ in a vacuum concentrator. Finally, the samples were frozen at $-80^{\circ} \mathrm{C}$, lyophilized and stored at $-20^{\circ} \mathrm{C}$ until spectroscopy measurement.

\section{NMR data acquisition and processing}

Prior to ${ }^{1} \mathrm{H}$ NMR analysis, the lyophilisates were resuspended in $20 \mathrm{mM}$ phosphate buffer ( $\mathrm{pH} 7.0$ ) containing $10 \% \mathrm{D}_{2} \mathrm{O}$ and 3-(Trimethylsilyl) propionic acid (TSP; Lancaster Synthesis, USA) as an internal standard as described previously ${ }^{70}$.

One-dimensional ${ }^{1} \mathrm{H}$ NMR spectra were acquired with a Bruker AVANCE III HD 700 spectrometer (Bruker, USA) equipped with a $5 \mathrm{~mm} \mathrm{HCN}$ TCI cryo-probe operating at $700 \mathrm{MHz}$ (16.4 Tesla). The ${ }^{1} \mathrm{H}$ NMR data were obtained using excitation sculpting for water suppressing and the following acquisition parameters: $25^{\circ} \mathrm{C}$ sample temperature, $9800 \mathrm{~Hz}$ sweep width, 256 transients with $32 \mathrm{~K}$ timedomain data points were accumulated with a repetition time of $3.2 \mathrm{~s}$ as previously described ${ }^{70}$.

Mestrenova version 8.0.1-10878 (Mestrelab Research S. L., Spain) software was used to process and analyze the ${ }^{1} \mathrm{H}$ NMR spectra. Equal concentrations of TSP in each sample were used as an internal standard for normalization. The figures show ${ }^{1} \mathrm{H}$ NMR data from a minimum of three independent experiments presented as mean \pm standard deviation (SD) and statistical significance was calculated with unpaired Student's $t$ tests. A $p$ value below 0.05 was considered significant.

\section{Cell viability, apoptosis, and cell cycle assays}

Cell viability was assessed as described previously ${ }^{70}$. In brief, the cell number was adjusted to 20,000 cells $/ \mathrm{ml}$ and triplicates of $100 \mu \mathrm{l}$ were plated per 96-well. For GLSi treatment, we plated the cells in neurosphere medium containing various drug concentrations $(1,5,10 \mu \mathrm{M}$ for C968 and 0.1, 0.5, 1.0 $\mu \mathrm{M}$ for CB839) or vehicle (DMSO). For the rescue experiments cells were treated with $10 \mu \mathrm{M}$ C968, $1 \mu \mathrm{M}$ CB839, or equal volumes of DMSO and either $4 \mathrm{mM}$ Glu (Sigma, \#G1251-100G) or $4 \mathrm{mM}$ aKG (Sigma, \#7589-25G) were added to the different conditions. The viable cell mass was assessed using the CellTiter-Blue ${ }^{\circledR}$ Cell Viability Assay (Promega, \#G8081) or Thiazolyl Blue Tetrazolium Bromide (MTT) (Sigma, \#2128-1G) according to the manufacturer's instructions. For CellTiter-Blue ${ }^{\circledR}$ the fluorescence was measured at $560 \mathrm{ex} / 590 \mathrm{em}$ and for MTT absorbance it was measured at $570 \mathrm{~nm}$ (reference $650 \mathrm{~nm}$ ) using a Safire 2 multiplate reader (Tecan, Switzerland). Biological replicates analyzed in Fig. 2: $n=4$ for NSC, JHH520, GBM1, 407 (C968), 268 (C968), and SF188 (C968); $n=3$ for 233, 407 (CB839), 268 (CB839), and SF188 (CB839).

Apoptosis induction was measured with the "Muse ${ }^{\circledR}$ Annexin V and Dead Cell Assay Kit" (Merck Millipore, USA). Therefore, GSCs were cultured in medium containing $10 \mu \mathrm{M} \mathrm{C} 968,1 \mu \mathrm{M}$ CB839, or vehicle (DMSO) for $48 \mathrm{~h}$, stained for Annexin $\mathrm{V}$ and 7-AAD according to the manufacturer's instructions, and flow cytometry measurements were performed on a Muse $^{\circledR}$ cell analyzer 
(Merck Millipore) as described in the manufacturer's instructions.

The cell cycle was analyzed with the "Muse ${ }^{\circledR}$ Cell Cycle Assay Kit" (Merck Millipore). Cells were treated with $10 \mu \mathrm{M}$ C968, $1 \mu \mathrm{M} \mathrm{CB839,}$ or vehicle (DMSO) for $48 \mathrm{~h}$, fixed with ice cold $70 \%$ ethanol at $-20^{\circ} \mathrm{C}$ for at least three hours and the DNA content was stained according to the manufacturers' instructions. Flow cytometry measurements were performed on a Muse ${ }^{\circledast}$ cell analyzer (Merck Millipore).

\section{Clonogenicity assays}

The clonogenicity of GSCs was assessed with colony forming assays in semi-solid agarose medium as described previously ${ }^{70}$. In brief, six-well plates were coated with $1.5 \mathrm{ml}$ of $1 \%$ agarose (Gibco, \#18300012) in pre-warmed neurosphere medium. After $1 \mathrm{~h}$ incubation at RT, $2 \mathrm{ml}$ of a single-cell suspension (3000 cells/well) in $0.6 \%$ agarose in neurosphere medium was added. After $1 \mathrm{~h}$ incubation at room temperature, $2 \mathrm{ml}$ neurosphere medium was added as a top layer. To test the effect of C968 and CB839 on GSC clonogenicity, we either added drugs $(10 \mu \mathrm{M}$ C968 or $1 \mu \mathrm{M}$ CB839) or equal volumes of vehicle (DMSO) to the upper medium layer. Twice a week the top layer was removed and $2 \mathrm{ml}$ fresh medium (with drug or vehicle) was added. After 3 weeks the top layer was removed, replaced by $1 \mathrm{ml}$ of $1 \mathrm{mg} / \mathrm{ml} \mathrm{4-Nitro} \mathrm{blue} \mathrm{tet-}$ razolium chloride (NBT) (Sigma, \#11383213001) in PBS and incubated overnight at $37^{\circ} \mathrm{C}$. The stained colonies were counted using the Clono Counter software ${ }^{74}$. Figure 3 includes the analysis of three biological replicates $(n=3)$ for all cell lines but NSCs. Due to the observed increased degree of biological variations between the replicates, we performed the assay with this cell line more often $(n=4$ for C968, $n=6$ for CB839).

\section{DCFDA ROS assay and GSH/GSSG ratio detection}

Accumulation of ROS was measured using $2^{\prime}, 7^{\prime}$ Dichlorofluorescin diacetate (DCFDA) (Sigma, \#D6883). Briefly, JHH520, GBM1, 407, and 233 cells were washed with PBS once and resuspended in PBS containing $50 \mu \mathrm{M}$ DCFDA. After incubation of $30 \mathrm{~min}$ at $37^{\circ} \mathrm{C}$, cells were again washed with $\mathrm{PBS}$, resuspended in neurosphere medium, and $3 \times 10^{5}$ cells per condition were transferred into flasks containing either drugs $(10 \mu \mathrm{M}$ C968 or $10 \mu \mathrm{M}$ CB839) or equal volumes of vehicle (DMSO). The cells were incubated for 24 or $48 \mathrm{~h}$ at $37^{\circ} \mathrm{C}$ under standard cell culture conditions. As a negative control, cells were incubated with $3 \mathrm{mM}$ of the ROS inhibitor NAC for $24 \mathrm{~h}$, as a positive control, cells were incubated with $500 \mu \mathrm{M}$ $\mathrm{H}_{2} \mathrm{O}_{2}$ for $30 \mathrm{~min}$. After 24 or $48 \mathrm{~h}$ the cells were washed once with PBS and resuspended in $700 \mu \mathrm{l}$ PBS. For every condition, $200 \mu \mathrm{l}$ triplicates of each condition were transferred into 96-well microplates. Fluorescence was measured at $493 \mathrm{em} / 515 \mathrm{ex}$ on a Safire 2 multiplate reader (Tecan).

For the quantification of the total reduced GSH and GSSG content we used the "GSH/GSSG Ratio Detection Assay Kit" (abcam, \#ab138881) according to the manufacturer's instructions. In brief, GSC cultures were treated with either $10 \mu$ M C968 or $1 \mu$ M CB839 for $48 \mathrm{~h}$. Subsequently, the total protein content was measured using the "DC ${ }^{\mathrm{TM}}$ Protein Assay Kit II" (BioRad, \#5000112) according to the manufacturer's instructions. The cells were lysed in $0.5 \%$ NP40 in PBS, centrifuged for $15 \mathrm{~min}$ at 13,000 rpm and $4{ }^{\circ} \mathrm{C}$, and the supernatant was used in a deproteinization reaction. Therefore, proteins were precipitated with $4 \mathrm{M}$ phosphochloric acid diluted to a final concentration of $1 \mathrm{M}$ within the cell lysate. After $2 \mathrm{~min}$ of centrifugation at $13,000 \mathrm{rpm}$ at $4{ }^{\circ} \mathrm{C}$, the cleared supernatant was then neutralized to $\mathrm{pH} 4-6$ by addition of $2 \mathrm{M}$ $\mathrm{KOH}$. This deproteinized lysate was then analyzed with the above mentioned GSH/GSSG Ratio Detection Assay Kit. The detected reduced and oxidized GSH (nmol) was then normalized to the total protein content $(\mathrm{mg})$ of the respective samples.

\section{RNA sequencing data from IVY Glioblastoma Project}

As described previously ${ }^{70}$ RNA sequencing data were generated from anatomic structures isolated by laser microdissection. Five tumor structures (leading edge $n=$ 19, infiltrating tumor $n=25$, cellular tumor $n=112$, perinecrotic zone $n=27$, and pseudopalisading cells around necrosis $n=41$ ) were identified by H\&E staining and compared with hyperplastic blood vessels $(n=23)$ and the microvascular proliferative region $(n=29)$. A total of 122 RNA samples were generated from 10 tumors and used for sequencing. The data were retrieved in March 2018. Website: (2015 Allen Institute for Brain Science. Ivy Glioblastoma Atlas Project [Internet]. Available from: glioblastoma.alleninstitute.org.

\section{Statistical analyses}

All statistics were performed with GraphPad Prism Software Version 8.0.2 (GraphPad Software Inc., USA). All results are presented as mean \pm SD from a minimum of at least three independent biological replicates. To calculate statistical significance in an experiment with two conditions (treated vs. untreated) we performed twotailed $t$-tests. When more than two conditions were compared with each other (mRNA expression data) we performed one-way ANOVA analyses and Bonferroni's tests for multiple comparisons. If applicable, normal distribution was confirmed with the Shapiro-Wilk method. For all experiments, significance was defined as a $p$ value below 0.05 . 


\section{Acknowledgements}

The authors thank Maria Stella Carro and Oliver Schnell (University Hospital Freiburg i. Br.) for generating and providing GSC 23, 233, 268, 349, and 407. The authors thank Guido Reifenberger and Gabriel Leprivier and their teams (Department of Neuropathology, University Medical Center Duesseldorf) for their support. The authors acknowledge access to the Juelich-Duesseldorf Biomolecular NMR Center that is jointly run by Forschungszentrum Juelich and Heinrich-Heine-Universitaet Duesseldorf. The authors thank Kevin Bochinsky for technical assistance with spectra acquisition. The authors thank Dieter Haeussinger (Department of Gastroenterology, Hepatology and Infectious Diseases, University Medical Center Duesseldorf) for supplying the GLS antibody. The authors thank Nadine Teichweyde (IUF Duesseldorf) for technical assistance. K.K. and J.T. were partially funded as a scholars of the Duesseldorf School of Oncology (DSO) of HHU University. The work has been co-financed by the SFF Grants of the HHU University, Duesseldorf, Germany, awarded to J.M. and U.D.K. The work of U.D.K. is supported by the Bundesministerium fuer Bildung und Forschung [03VP03791], the Volkswagen Stiftung, the Hempel Family Foundation and the Brigitte-and Dr. KonstanzeWegener Foundation. R.A.B. is supported by an NIHR funded Biomedical Research Centre in Cambridge and is also an NIHR Senior Investigator.

\section{Author details}

${ }^{1}$ Neurosurgery Department, University Hospital Duesseldorf, 40225

Duesseldorf, Germany. ${ }^{2}$ Institute of Complex Systems (ICS-6) Structural Biochemistry and JuStruct: Juelich Center for Structural Biology,

Forschungszentrum Juelich, 52425 Juelich, Germany. ${ }^{3}$ John van Geest Centre for Brain Repair and WT/MRC Cambridge Stem Cell Institute, Department of Clinical Neurosciences, University of Cambridge, CB2 OPY Cambridge, UK. ${ }^{4}$ Institut für Physikalische Biologie, Heinrich Heine University Duesseldorf, 40225 Duesseldorf, Germany. ${ }^{5}$ Neurosurgery Department, University Hospital Bonn, 53127 Bonn, Germany. ${ }^{6}$ German Cancer Consortium (DKTK), Essen/ Duesseldorf, Germany

\section{Conflict of interest}

The authors declare that they have no conflict of interest.

\section{Publisher's note}

Springer Nature remains neutral with regard to jurisdictional claims in published maps and institutional affiliations.

The online version of this article (https://doi.org/10.1038/s41420-020-0258-3) contains supplementary material, which is available to authorized users.

\section{Received: 12 December 2019 Revised: 27 February 2020 Accepted: 26 March 2020 \\ Published online: 16 April 2020}

\section{References}

1. Hanahan, D. \& Weinberg, R. A. Hallmarks of cancer: the next generation. Cell 144, 646-674 (2011).

2. DeBerardinis, R. J. \& Cheng, T. Q's next: the diverse functions of glutamine in metabolism, cell biology and cancer. Oncogene 29, 313-324 (2010).

3. Gao, P. et al. c-Myc suppression of miR-23a/b enhances mitochondrial glutaminase expression and glutamine metabolism. Nature 458, 762-765 (2009).

4. Wise, D. R. et al. Myc regulates a transcriptional program that stimulates mitochondrial glutaminolysis and leads to glutamine addiction. Proc. Natl Acad. Sci. USA 105, 18782-18787 (2008).

5. Donohoe, D. R. \& Bultman, S. J. Metaboloepigenetics: interrelationships between energy metabolism and epigenetic control of gene expression. J. Cell. Physiol. 227, 3169-3177 (2012).

6. Yun, J., Johnson, J. L., Hanigan, C. L. \& Locasale, J. W. Interactions between epigenetics and metabolism in cancers. Front. Oncol. 2, 163 (2012).

7. Pelicano, H., Martin, D. S., Xu, R. H. \& Huang, P. Glycolysis inhibition for anticancer treatment. Oncogene 25, 4633-4646 (2006).

8. Galluzzi, L., Kepp, O., Vander Heiden, M. G. \& Kroemer, G. Metabolic targets for cancer therapy. Nat. Rev. Drug Discov. 12, 829-846 (2013).
9. Wang, J. B. et al. Targeting mitochondrial glutaminase activity inhibits oncogenic transformation. Cancer Cell 18, 207-219 (2010).

10. DeBerardinis, R. J., Lum, J. J., Hatzivassiliou, G. \& Thompson, C. B. The biology of cancer: metabolic reprogramming fuels cell growth and proliferation. Cell Metab. 7, 11-20 (2008).

11. Wise, D. R. \& Thompson, C. B. Glutamine addiction: a new therapeutic target in cancer. Trends Biochem. Sci. 35, 427-433 (2010).

12. Altman, B. J., Stine, Z. E. \& Dang, C. V. From Krebs to clinic: glutamine metabolism to cancer therapy. Nat. Rev. Cancer 16, 619-634 (2016).

13. McBrayer, S. K. et al. Transaminase inhibition by 2-hydroxyglutarate impairs glutamate biosynthesis and redox homeostasis in glioma. Cell 175, 101-116 e125 (2018).

14. Gross, M. I. et al. Antitumor activity of the glutaminase inhibitor CB-839 in triple-negative breast cancer. Mol. Cancer Ther. 13, 890-901 (2014).

15. Guo, L. et al. Blockage of glutaminolysis enhances the sensitivity of ovarian cancer cells to PI3K/mTOR inhibition involvement of STAT3 signaling. Tumour Biol. 37, 11007-11015 (2016).

16. Zacharias, N. M. et al. Metabolic differences in glutamine utilization lead to metabolic vulnerabilities in prostate cancer. Sci. Rep. 7, 16159 (2017).

17. Boysen, G. et al. Glutaminase inhibitor CB-839 increases radiation sensitivity of lung tumor cells and human lung tumor xenografts in mice. Int. J. Radiat. Biol. 1-23, https://doi.org/10.1080/09553002.2018.1558299 (2018).

18. Xie, C. et al. Inhibition of mitochondrial glutaminase activity reverses acquired erlotinib resistance in non-small cell lung cancer. Oncotarget 7, 610-621 (2016).

19. Han, T. et al. A novel glutaminase inhibitor-968 inhibits the migration and proliferation of non-small cell lung cancer cells by targeting EGFR/ERK signaling pathway. Oncotarget 8, 28063-28073 (2017).

20. Wang, D. et al. The glutaminase-1 inhibitor 968 enhances dihydroartemisininmediated antitumor efficacy in hepatocellular carcinoma cells. PLOS ONE 11, e0166423 (2016).

21. Kim, J. H. et al. Effects of metformin on colorectal cancer stem cells depend on alterations in glutamine metabolism. Sci. Rep. 8, 409 (2018).

22. Yuan, L. et al. Glutaminase inhibitor compound 968 inhibits cell proliferation and sensitizes paclitaxel in ovarian cancer. Am. J. Transl. Res. 8, 4265-4277 (2016).

23. Li, B. et al. Targeting glutaminase 1 attenuates stemness properties in hepatocellular carcinoma by increasing reactive oxygen species and suppressing Wnt/beta-catenin pathway. EBioMedicine 39, 239-254 (2019).

24. Panosyan, E. H. et al. Clinical aggressiveness of malignant gliomas is linked to augmented metabolism of amino acids. J. Neuro-Oncol. 128, 57-66 (2016).

25. Cheng, T. et al. Pyruvate carboxylase is required for glutamine-independent growth of tumor cells. Proc. Natl Acad. Sci. USA 108, 8674-8679 (2011).

26. Tanaka, K. et al. Compensatory glutamine metabolism promotes glioblastoma resistance to mTOR inhibitor treatment. J. Clin. Investig. 125, 1591-1602 (2015).

27. Tardito, S. et al. Glutamine synthetase activity fuels nucleotide biosynthesis and supports growth of glutamine-restricted glioblastoma. Nat. Cell Biol. 17, 1556-1568 (2015).

28. Cheng, L. et al. Elevated invasive potential of glioblastoma stem cells. Biochem. Biophys. Res. Commun. 406, 643-648 (2011).

29. Bao, S. et al. Glioma stem cells promote radioresistance by preferential activation of the DNA damage response. Nature 444, 756-760 (2006).

30. Liu, G. et al. Analysis of gene expression and chemoresistance of CD133+ cancer stem cells in glioblastoma. Mol. Cancer 5, 67 (2006).

31. Singh, S. K. et al. Identification of a cancer stem cell in human brain tumors. Cancer Res. 63, 5821-5828 (2003).

32. Kahlert, U. D., Mooney, S. M., Natsumeda, M., Steiger, H. J. \& Maciaczyk, J. Targeting cancer stem-like cells in glioblastoma and colorectal cancer through metabolic pathways. Int. J. Cancer 140, 10-22 (2017).

33. Chinnaiyan, P. et al. The metabolomic signature of malignant glioma reflects accelerated anabolic metabolism. Cancer Res. 72, 5878-5888 (2012).

34. DeBerardinis, R. J. \& Chandel, N. S. Fundamentals of cancer metabolism. Sci. Adv. 2, e1600200 (2016).

35. Siebzehnrubl, F. A. et al. The ZEB1 pathway links glioblastoma initiation, invasion and chemoresistance. EMBO Mol. Med. 5, 1196-1212 (2013).

36. Cao, J. et al. Expression of GLS1 in intrahepatic cholangiocarcinoma and its clinical significance. Mol. Med Rep. 20, 1915-1924 (2019).

37. Takaoka, Y. et al. Mitochondrial pyruvate carrier 1 expression controls cancer epithelial-mesenchymal transition and radioresistance. Cancer Sci. 110 1331-1339 (2019) 
38. Mao, X. G. et al. LIN28A facilitates the transformation of human neural stem cells and promotes glioblastoma tumorigenesis through a pro-invasive genetic program. Oncotarget 4, 1050-1064 (2013).

39. Maciaczyk, J., Singec, I., Maciaczyk, D., Klein, A. \& Nikkhah, G. Restricted spontaneous in vitro differentiation and region-specific migration of long-term expanded fetal human neural precursor cells after transplantation into the adult rat brain. Stem Cells Dev. 18, 1043-1058 (2009).

40. Yoo, S. \& Bieda, M. C. Differences among brain tumor stem cell types and fetal neural stem cells in focal regions of histone modifications and DNA methylation, broad regions of modifications, and bivalent promoters. BMC Genom 15, 724 (2014).

41. Feve, M. et al. Comparative expression study of the endo-G protein coupled receptor (GPCR) repertoire in human glioblastoma cancer stem-like cells, U87MG cells and non malignant cells of neural origin unveils new potential therapeutic targets. PLOS ONE 9, e91519 (2014).

42. Xi, J. et al. GLS1 promotes proliferation in hepatocellular carcinoma cells via AKT/GSK3beta/CyclinD1 pathway. Exp. Cell Res. 381, 1-9 (2019).

43. Zhang, J. et al. Inhibition of GLS suppresses proliferation and promotes apoptosis in prostate cancer. Biosci. Rep. 39, https://doi.org/10.1042/ BSR20181826 (2019).

44. Agostini, M. et al. Metabolic reprogramming during neuronal differentiation. Cell Death Differ. 23, 1502-1514 (2016).

45. Wang, Y., Huang, Y., Zhao, L., Li, Y. \& Zheng, J. Glutaminase 1 is essential for the differentiation, proliferation, and survival of human neural progenitor cells. Stem cells Dev. 23, 2782-2790 (2014).

46. Kahlert, U. D. et al. ZEB1 promotes invasion in human fetal neural stem cells and hypoxic glioma neurospheres. Brain Pathol. 25, 724-732 (2015).

47. Kahlert, U. D. et al. CD133/CD15 defines distinct cell subpopulations with differential in vitro clonogenic activity and stem cell-related gene expression profile in in vitro propagated glioblastoma multiforme-derived cell line with a PNET-like component. Folia Neuropathol. 50, 357-368 (2012).

48. Singh, S. K. et al. Identification of human brain tumour initiating cells. Nature 432, 396-401 (2004).

49. Owen, J. B. \& Butterfield, D. A. Measurement of oxidized/reduced glutathione ratio. Methods Mol. Biol. 648, 269-277 (2010).

50. Grinde, M. T. et al. Glutamine to proline conversion is associated with response to glutaminase inhibition in breast cancer. Breast Cancer Res. 21, 61 (2019).

51. Marin-Valencia, I. et al. Analysis of tumor metabolism reveals mitochondrial glucose oxidation in genetically diverse human glioblastomas in the mouse brain in vivo. Cell Metab. 15, 827-837 (2012).

52. Janzer, A. et al. Metformin and phenformin deplete tricarboxylic acid cycle and glycolytic intermediates during cell transformation and NTPs in cancer stem cells. Proc. Natl Acad. Sci. USA 111, 10574-10579 (2014).

53. Sato, M. et al. Spheroid cancer stem cells display reprogrammed metabolism and obtain energy by actively running the tricarboxylic acid (TCA) cycle. Oncotarget 7, 33297-33305 (2016).

54. Foster, D. A., Yellen, P., Xu, L. \& Saqcena, M. Regulation of G1 cell cycle progression: distinguishing the restriction point from a nutrient-sensing cell growth checkpoint(s). Genes Cancer 1, 1124-1131 (2010).

55. Saqcena, M. et al. Amino acids and mTOR mediate distinct metabolic checkpoints in mammalian G1 cell cycle. PLoS ONE 8, e74157 (2013).
56. Kahlert, U. D. et al. Alterations in cellular metabolome after pharmacological inhibition of Notch in glioblastoma cells. Int. J. Cancer 138, 1246-1255 (2016).

57. Zhou, R. et al. [(18)F](2S,4R)4-Fluoroglutamine PET detects glutamine pool size changes in triple-negative breast cancer in response to glutaminase inhibition. Cancer Res. 77, 1476-1484 (2017).

58. Boysen, G. et al. Glutaminase inhibitor CB-839 increases radiation sensitivity of lung tumor cells and human lung tumor xenografts in mice. Int. J. Radiat. Biol. 95, 436-442 (2019).

59. Lin, A. et al. Off-target toxicity is a common mechanism of action of cancer drugs undergoing clinical trials. Sci. Transl. Med. 11, https://doi.org/10.1126/ scitransImed.aaw8412 (2019).

60. Daemen, A. et al. Pan-cancer metabolic signature predicts co-dependency on glutaminase and de novo glutathione synthesis linked to a highmesenchymal cell state. Cell Metab. 28, 383-399 e389 (2018).

61. Zhou, R. et al. Glutamate-weighted chemical exchange saturation transfer magnetic resonance imaging detects glutaminase inhibition in a mouse model of triple-negative breast cancer. Cancer Res. 78, 5521-5526 (2018).

62. Li, J. et al. Heat shock factor 1 epigenetically stimulates glutaminase-1dependent mTOR activation to promote colorectal carcinogenesis. Mol. Ther. 26, 1828-1839 (2018).

63. Yu, Y. et al. Targeting glutaminase-mediated glutamine dependence in papillary thyroid cancer. J. Mol. Med. 96, 777-790 (2018).

64. Yu, Z. et al. NVP-BEZ235, a novel dual PI3K-mTOR inhibitor displays anti-glioma activity and reduces chemoresistance to temozolomide in human glioma cells. Cancer Lett. 367, 58-68 (2015).

65. Mecca, C. et al. PP242 counteracts glioblastoma cell proliferation, migration, invasiveness and stemness properties by inhibiting mTORC2/AKT. Front. Cell Neurosci. 12, 99 (2018).

66. Liao, J. et al. Regulation of stem-like cancer cells by glutamine through betacatenin pathway mediated by redox signaling. Mol. Cancer 16, 51 (2017).

67. Martin-Rufian, M. et al. Both GLS silencing and GLS2 overexpression synergize with oxidative stress against proliferation of glioma cells. J. Mol. Med. 92, 277-290 (2014).

68. Paananen, J. \& Fortino, V. An omics perspective on drug target discovery platforms. Brief Bioinform. https://doi.org/10.1093/bib/bbz122 (2019).

69. Giesen, B. et al. Influence of synthesis methods on the internalization of fluorescent gold nanoparticles into glioblastoma stem-like cells. J. Inorg. Biochem. 203, 110952 (2020).

70. Koch, K. et al. Reciprocal regulation of the cholinic phenotype and epithelialmesenchymal transition in glioblastoma cells. Oncotarget 7, 73414-73431 (2016).

71. Schneider, C. A., Rasband, W. S. \& Eliceiri, K. W. NIH Image to ImageJ: 25 years of image analysis. Nat. Methods 9, 671-675 (2012).

72. Glunde, K., Jie, C. \& Bhujwalla, Z. M. Molecular causes of the aberrant choline phospholipid metabolism in breast cancer. Cancer Res. 64, 4270-4276 (2004).

73. Tyagi, R. K., Azrad, A., Degani, H. \& Salomon, Y. Simultaneous extraction of cellular lipids and water-soluble metabolites: evaluation by NMR spectroscopy. Magn. Reson. Med. 35, 194-200 (1996).

74. Niyazi, M., Niyazi, I. \& Belka, C. Counting colonies of clonogenic assays by using densitometric software. Radiat. Oncol. 2, 4 (2007). 